\title{
The Autobots are Here to Stay: Robotics in Medicine
}

All of us are familiar with the Autobots. They are one of the primary factions in the transformers mythos. They usually find themselves defending both their own race and other species against the decepticons and upholding justice and freedom throughout the galaxy. Enter the Robots into the world of medicine. Medicine as a field has been growing by leaps and bounds. What sounded as a myth once is being converted into reality.

A medical robot is a robot that allows surgeons greater access to areas under operation using more precise and less invasive methods. They are in most telemanipulators, which use the surgeon's actions on one side to control the 'effector' on the other side.

Various types of medical robots are as follows:

- Surgical robots: These robots either allow surgical operations to be carried out with greater precision than an unaided human surgeon or allow remote surgery where a human surgeon is not physically present with the patient.

- Rehabilitation robots: This group facilitates and supports the lives of infirm, elderly people, or those with dysfunction of body parts effecting movement. These robots are also used for rehabilitation and related procedures, such as training and therapy.

- Biorobots: A group of robots designed to imitate the cognition of humans and animals.

- Telepresence robots: Allow off-site medical professionals to move, look around, communicate and participate from remote locations.

- Pharmacy automation: Robotic systems to dispense oral solids in a retail pharmacy setting or preparing sterile IV admixtures in a hospital pharmacy setting.

- Disinfection robot has the capability to disinfect a whole room in mere minutes, generally using ultraviolet light technology. There are being used to fight Ebola virus disease 4.

Postgraduate Institute of Medical Education and Research, has acquired the da Vinci system. It is not an automated surgical system. Rather, it is a remote controlled by a human surgeon. The word 'remote' might be a bit misleading; though, while the system is capable of being operated from just about anywhere, it is most common to have the surgeon sitting right next to it, in the operating room with the patient. The benefit of the da Vinci system is not really the potential for remote access, instead, it is that the surgeon can use tiny robotic tools that mean a much smaller incision, along with visual enhancements like infrared imaging that provide valuable information than their eyes might not.

As we leap forward into the advance domain of medicine, we hope the da Vinci becomes our autobot ... helping us to reach newer heights in medicine.

Ramandeep S Virk

Associate Professor

Department of ENT and Head and Neck Surgery

Postgraduate Institute of Medical Education and

Research, Chandigarh, India 